\title{
A numerical solution for plain problems of theory of elasticity
}

\author{
Radik Gabbasov $^{1}$, Michael Gandzhuntsev ${ }^{1}$, Vladimir Filatov ${ }^{1}$, Dzhurakulov Muniev² ${ }^{2}$ and Alaa \\ El-Din Mansour,"*
}

${ }^{1}$ Moscow State University of Civil Engineering, Yaroslavskoeshosse, 26, Moscow, 129337, Russia

${ }^{2}$ Tajik Technical University, Rudaki str., 17, Dushanbe, 734061, Tajikistan

\begin{abstract}
In this paper, a generation of a numerical algorithm for solving theory of elasticity plain problem concerning displacements is desired ; using difference equations that the method of successive approximations proceed through transforming a complex (to be solved) differential equation into an algebraic one that's much more easier to solve. The algebraic equations resulted adopt both positioned nodes; interior nodes of a computational grid, and contour (boundary) nodes; including corner ones. The evaluation of the new generated algorithm is being validated through applying it on solving a problem of a laterally fixed deep-beam. Obtained numerical results out of the new algorithm to be compared with those early known and stated in the references listed at the last part of the article.
\end{abstract}

\section{Introduction}

The differential equations of successive approximations method [1] are applied to the plain problem of elasticity theory in terms of stresses such as that presented in Equation 1.

$$
\alpha \frac{\partial^{2} u}{\partial \xi^{2}}+\beta \frac{\partial^{2} v}{\partial \xi \partial \eta}+\gamma \frac{\partial^{2} u}{\partial \eta^{2}}=0
$$

The presented scientific work targets further development of forms of those equations considering its application on elasticity theory problems where unknowns will be featured out as displacement $\mathrm{s} u$ and $v$.

Equation2 in its dimensionless form demonstrates that as follows:

$$
\gamma \frac{\partial^{2} v}{\partial \xi^{2}}+\beta \frac{\partial^{2} u}{\partial \xi \partial \eta}+\alpha \frac{\partial^{2} v}{\partial \eta^{2}}=0
$$

Where $\xi=\frac{x}{a} ; \eta=\frac{y}{a} ; u=\frac{U}{a} ; v=\frac{V}{a} ;$ For plain stress: $\alpha=1 ; \beta=\frac{1+\mu}{2} ; \gamma=\frac{1-\mu}{2}$;

*Corresponding author: alaa_for_all@hotmail.com 
For plain strain: $\alpha=1 ; \beta=\frac{1+\mu_{1}}{2} ; \gamma=\frac{1-\mu_{1}}{2} ; \mu_{1}=\frac{\mu}{1-\mu}$;Where $\alpha$ - a characteristic size; $\mu$ Poisson's ratio.

\section{In Details}

Obtaining the approximated differential Equation 2, a down stated difference terming of Equation 1 could be written as Equation 3 [2]:

$$
\begin{aligned}
& (c \alpha+b \gamma) u_{i-1, j-1}+2(5 c \alpha-b \gamma) u_{i-1, j}+(c \alpha+b \gamma) u_{i-1, j+1}- \\
& -2(c \alpha-5 b \gamma) u_{i, j-1}-20(c \alpha+b \gamma) u_{i, j}-2(c \alpha-5 b \gamma) u_{i, j+1}+ \\
& +(c \alpha+b \gamma) u_{i+1, j-1}+2(5 c \alpha-b \gamma) u_{i+1, j}+(c \alpha+b \gamma) u_{i+1, j+1}+{ }^{+} \\
& +3 \beta\left(v_{i-1, j-1}-v_{i-1, j+1}-v_{i+1, j-1}+v_{i+1, j+1}\right)=0
\end{aligned}
$$

Similar way, we can obtainEquation 3 for $\mathrm{v}$ approximating the differential Equation 2; Replacing its terms $\mathrm{u}, \mathrm{v}, \alpha, \gamma$, respectively with $\mathrm{v}, \mathrm{u}, \gamma, \alpha$.

This above generated pair of equations Equation 3 and Equation 3'; enables us to calculate $\mathrm{u}$ and $\mathrm{v}$, if the contour (boundary or edge) displacements are specified. If stresses are specified on sides contour nodes (points), additional differential equations for other edges (upper \& lower) and corner nodes are necessary to be obtained as well, so; let us write the relation between the stresses and displacements (in its dimensionless form) for plane stresses case as follows:

$$
\begin{gathered}
\sigma^{\xi}=\frac{1}{1-\mu^{2}}\left(\frac{\partial u}{\partial \xi}+\mu \frac{\partial v}{\partial \eta}\right) \\
\sigma^{\eta}=\frac{1}{1-\mu^{2}}\left(\mu \frac{\partial u}{\partial \xi}+\frac{\partial v}{\partial \eta}\right) ; \\
\tau^{\xi \eta}=\frac{1}{2(1+\mu)}\left(\frac{\partial u}{\partial \eta}+\mu \frac{\partial v}{\partial \xi}\right),
\end{gathered}
$$

Where $\sigma^{\xi}=\frac{\sigma_{x}}{E} ; \sigma^{\eta}=\frac{\sigma_{y}}{E} ; \tau^{\xi \eta}=\frac{\tau_{x y}}{E}$.

The right hand sides of the equations set Equation4-6 represents the first derivatives of displacements. The differential expressions for these derivatives are obtained the same fashion as Equation 3. Consequently, for upper edge nodes: 


$$
\begin{gathered}
\left(\frac{\partial u}{\partial \xi}\right)_{i, j}=\frac{1}{4 h(5 c \alpha+b \gamma)}\left[(c \alpha+7 b \gamma) u_{i, j-1}-20(c \alpha+b \gamma) u_{i, j}+\right. \\
+(c \alpha+7 b \gamma) u_{i, j+1}-2(c \alpha-3 b \gamma) u_{i+1, j-1}+4(5 c \alpha-b \gamma) u_{i+1, j}- \\
-2(c \alpha-3 b \gamma) u_{i+1, j+1}+(c \alpha-b \gamma) u_{i+2, j-1}+(c \alpha-b \gamma) u_{i+2, j+1}+ \\
\left.+\beta\left(7 v_{i, j-1}-7 v_{i, j+1}-8 v_{i+1, j-1}+8 v_{i+1, j+1}+v_{i+2, j-1}-v_{i+2, j+1}\right)\right] \\
\left(\frac{\partial u}{\partial \eta}\right)_{i, j}=\frac{1}{36 h \gamma}\left[-6(c \alpha+3 b \gamma)\left(u_{i, j-1}-u_{i, j+1}\right)+12 c \alpha\left(u_{i+1, j-1}-u_{i+1, j+1}\right)-\right. \\
-6 c \alpha\left(u_{i+2, j-1}-u_{i+2, j+1}\right)-\beta\left(13 v_{i, j-1}-26 v_{i, j}+13 v_{i, j+1}-\right. \\
\left.\left.-14 v_{i+1, j-1}+28 v_{i+1, j}-14 v_{i+1, j+1}+v_{i+2, j-1}-2 v_{i+2, j}+v_{i+2, j+1}\right)\right]
\end{gathered}
$$

In the equations Equation 3, Equation 7 and Equation $8 ; c=\frac{\tau}{h} ; b=\frac{h}{\tau}$.

For $\frac{\partial v}{\partial \xi}$ and $\frac{\partial v}{\partial \eta}$, the equations generated the same way as Equation7 and Equation 8; replacing $u$ with $v, v$ with $u, \alpha$ with $\gamma$ and $\gamma$ with $\alpha$.

For lower edge nodes; the above pair of equationsEquation7 and Equation 8is to be rewritten with appropriate replaced indices; thus, replacing $\frac{\partial u}{\partial \xi}, \frac{\partial v}{\partial \xi}$ and $\beta$.

For left edge nodes, differential expressions for the first derivatives of $u$ and $v$ are to be generated from upper edge equations, adopting the following indices changes of $u$ to $v, v$ to $u, \tau$ to $h, h$ to $\tau$ and finally $\xi$ to $\eta, \eta$ to $\xi$.

For right edge nodes, its equations are generated by proceeding necessary replacements of terms $\frac{\partial u}{\partial \xi}, \frac{\partial v}{\partial \xi}$ and $\beta$; the same way as before stated in left edge equations.

Consequently; the resulted equations of $u$ and $v$ derivatives together with the equations set Equation 4-6 are used to come out with the stress expressions for all edge nodes except for corner ones. Corner nodes equations can be driven as follows:

For upper-left corner node coordinated at $(i, j)$, a differential equation expressing $\frac{\partial u}{\partial \xi}$ could be reviewed as: $\frac{\partial u}{\partial \xi}$

$$
\begin{aligned}
\left(\frac{\partial u}{\partial \xi}\right)_{i, j} & =\frac{1}{72 \tau \alpha}\left[-(87 c \alpha-21 b \gamma) u_{i, j}+6(3 c \alpha-7 b \gamma) u_{i, j+1}-\right. \\
& -3(c \alpha-7 b \gamma) u_{i, j+2}+6(17 c \alpha+3 b \gamma) u_{i+1, j}-36(c \alpha+b \gamma) u_{i+1, j+1}+ \\
& +6(c \alpha+3 b \gamma) u_{i+1, j+2}-3(5 c \alpha+b \gamma) u_{i+2, j}+6(3 c \alpha+b \gamma) u_{i+2, j+1}- \\
& -3(c \alpha+b \gamma) u_{i+2, j+2}-\beta\left(-50 v_{i, j}+58 v_{i, j+1}-8 v_{i, j+2}+\right. \\
& \left.\left.+58 v_{i+1, j}-68 v_{i+1, j+1}+10 v_{i+1, j+2}-8 v_{i+2, j}+10 v_{i+2, j+1}-2 v_{i+2, j+2}\right)\right] .
\end{aligned}
$$

Other differential equations could be generated the same fashion.

For upper-right corner node, its equations are generated by proceeding replacements of the terms $\frac{\partial v}{\partial \eta}, \frac{\partial u}{\partial \eta}$ and $\beta$ same way as before mentioned in upper-left corner node equations, 
adopting necessary indices changes.

Substituting the values of $u$, vat first derivatives of corners' nodes in the right hand sides of expressions $\sigma^{\xi}+\sigma^{\xi \eta}$ and $\sigma^{\eta}+\sigma^{\xi \eta}$ [the Equations set Equation 4-6], we can obtain a couple of differential equations determining $u$ and $v$ at each corner node.

Differential expressions for stress at an internal node within the integration area, located at $(i, j)$ coordinates could be obtained the same way. The pair Equation 3, Equation7 shows how $\sigma_{i, j}^{\xi}$ and $\sigma_{i, j}^{\eta}$ tobe expressed:

$$
\begin{aligned}
\sigma_{i, j}^{\xi}=\frac{1}{1-\mu^{2}} & {\left[-B_{1} u_{i-1, j-1}-B_{2} u_{i-1, j}-B_{1} u_{i-1, j+1}+B_{1} u_{i+1, j-1}+\right.} \\
+ & B_{2} u_{i+1, j}+B_{1} u_{i+1, j+1}-B_{3}\left(v_{i-1, j-1}-v_{i-1, j+1}\right)+ \\
& \left.+B_{4}\left(v_{i, j-1}-v_{i, j+1}\right)-B_{3}\left(v_{i+1, j-1}-v_{i+1, j+1}\right)\right] \\
\sigma_{i, j}^{\eta}=\frac{1}{1-\mu^{2}}[ & -B_{5} u_{i-1, j-1}-B_{6} u_{i-1, j}-B_{5} u_{i-1, j+1}+B_{5} u_{i+1, j-1}+ \\
+ & B_{6} u_{i+1, j}+B_{5} u_{i+1, j+1}-B_{7}\left(v_{i-1, j-1}-v_{i-1, j+1}\right)+ \\
+ & \left.B_{8}\left(v_{i, j-1}-v_{i, j+1}\right)-B_{7}\left(v_{i+1, j-1}-v_{i+1, j+1}\right)\right]
\end{aligned}
$$

Where $B_{1}=\frac{b \gamma}{6 \tau \alpha}+\mu \frac{\beta}{6 h \alpha} ; B_{2}=\frac{3 c \alpha-2 b \gamma}{6 \tau \alpha}-\mu \frac{\beta}{3 h \alpha} ; B_{3}=\frac{\beta}{6 \tau \alpha}+\mu \frac{c \gamma}{6 h \alpha} ;$

$B_{4}=\frac{\beta}{3 \tau \alpha}+\mu \frac{2 c \alpha-3 b \gamma}{6 h \alpha} ; B_{5}=\mu \frac{b \gamma}{6 \tau \alpha}+\frac{\beta}{6 h \alpha} ; B_{6}=\mu \frac{3 c \alpha-2 b \gamma}{6 \tau \alpha}-\frac{\beta}{3 h \alpha} ;$

$B_{7}=\mu \frac{\beta}{6 \tau \alpha}+\frac{c \gamma}{6 h \alpha} ; B_{8}=\mu \frac{\beta}{3 \tau \alpha}+\frac{2 c \alpha-3 b \gamma}{6 h \alpha}$.

In means of evaluation for the pervious obtained innovated algorithm of equations; a problem of a deep-beam rigidly fixed on its right \& left sides (an unreleased moment connection) which is uniformly line - loaded on its top Figure 1 had been tested $[3,4]$ based on resulted deformations, stresses values that areshown in Table 1compared to other stresses values out of different solution methods ; considering $(\mu=1 / 6)$.

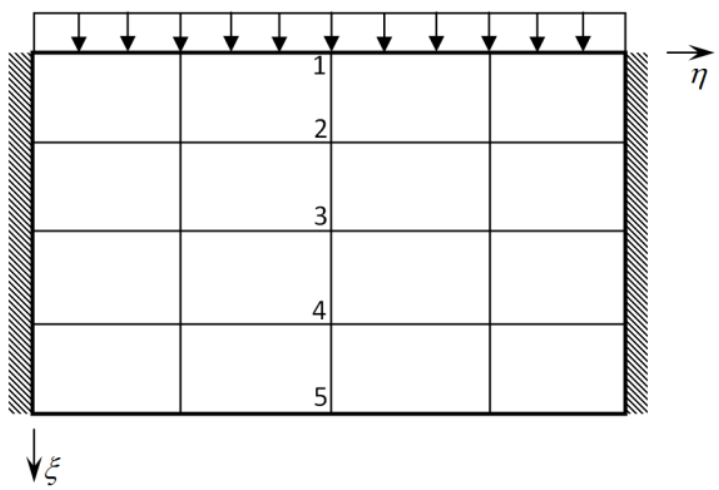

Fig. 1. Shows the nodes grid over the deep-beam problem understudy. 
Table 1. Different stresses values comparison.

\begin{tabular}{|c|c|c|c|c|c|c|c|c|c|c|}
\hline \multirow[t]{2}{*}{$\begin{array}{l}01 \\
0 \\
0 \\
0 \\
0\end{array}$} & \multicolumn{2}{|c|}{$\begin{array}{l}\text { Finite element } \\
\text { analysis - a grid } \\
\text { (plate) size of } \\
(6 \times 12) \text { nodes }\end{array}$} & \multicolumn{2}{|c|}{$\begin{array}{c}\text { Finite difference } \\
\text { - a grid (plate) } \\
\text { size of }(6 \times 12) \\
\text { nodes }\end{array}$} & \multicolumn{2}{|c|}{$\begin{array}{c}\text { successive } \\
\text { approximations } \\
\text { - a grid (plate) } \\
\text { size of }(8 \times 16) \\
\text { nodes }- \text { direct } \\
\text { stress } \\
\text { determination }\end{array}$} & \multicolumn{2}{|c|}{$\begin{array}{c}\text { successive } \\
\text { approximations } \\
\text { - a grid (plate) } \\
\text { size of }(8 \times 16) \\
\text { nodes }- \text { stress } \\
\text { determination } \\
\text { through } \\
\text { deformation }\end{array}$} & \multicolumn{2}{|c|}{$\begin{array}{c}\text { successive } \\
\text { approximations } \\
\text { - a grid (plate) } \\
\text { size of }(16 \times 32) \\
\text { nodes }- \text { stress } \\
\text { determination } \\
\text { through } \\
\text { deformation }\end{array}$} \\
\hline & $\sigma_{\eta}$ & $\sigma_{\xi}$ & $\sigma_{\eta}$ & $\sigma_{\xi}$ & $\sigma_{\eta}$ & $\sigma_{\xi}$ & $\sigma_{\eta}$ & $\sigma_{\xi}$ & $\sigma_{\eta}$ & $\sigma_{\xi}$ \\
\hline 5 & 0,268 & 0 & 0,299 & 0 & 0,313 & 0 & 0,330 & 0 & 0,317 & 0 \\
\hline 4 & 0,041 & $-0,146$ & 0,042 & $-0,151$ & 0,041 & $-0,148$ & 0,046 & $-0,145$ & 0,042 & $-0,148$ \\
\hline 3 & 0,013 & $-0,441$ & 0,010 & $-0,442$ & 0,010 & $-0,445$ & 0,011 & $-0,446$ & 0,010 & $-0,446$ \\
\hline 2 & $-0,104$ & $-0,804$ & $-0,103$ & $-0,798$ & $-0,105$ & $-0,805$ & $-0,113$ & $-0,810$ & $-0,109$ & $-0,806$ \\
\hline 1 & $-0,684$ & $-1,0$ & $-0,755$ & $-1,0$ & $-0,764$ & $-1,0$ & $-0,774$ & $-1,0$ & $-0,767$ & $-1,0$ \\
\hline
\end{tabular}

For nodes stresses along theloadededge, stresses are tabulated in Table 2.

Table 2. Loading line nodes stresses $[5,6]$.

\begin{tabular}{|l|l|l|}
\hline Method used for obtaining stresses & Grid size (nxm) & $\sigma_{\eta}$ \\
\hline Finite difference method & $6 \times 12$ & 1,935 \\
\hline \multirow{2}{*}{ Successive approximations method(direct stress determination) } & $4 \times 8$ & 2,168 \\
\cline { 2 - 3 } & $12 \times 94$ & 3,889 \\
\hline $\begin{array}{l}\text { Successive approximations method(stress determination through } \\
\text { deformation) }\end{array}$ & $8 \times 16$ & 3,611 \\
\cline { 2 - 3 } & $16 \times 32$ & 5,15 \\
\hline
\end{tabular}

\section{Conclusions}

The presented algorithm for solving the theory of elasticity plain problems through displacements could be considered reliable for calculating plain problems same as the tested one, also could be consideredmore effective in those cases when deep-beams or plates are; with holes (perforated), and loaded in-plane.

\section{References}

1. R.F. Gabbasov, Application of successive approximations for plain problems in theory of elasticity 23-26, 4 (1982)

2. E.Ginke, Shipbuilding 2, 274-296 (1994)

3. R.F. Gabbasov, A.R.Gabbassov,V.V. Filatov,Numerical construction of discontinuous problems of structural mechanics (Publ. h. DIA, Moscow, 2008)

4. S.P. Timoshenko, J. Goodier,Theory of elasticity (Publ. h. Science, Moscow, 1999)

5. N.N.Shaposhnikov, A.S.Volkov, Studies in the theory of structures 22, 134-146 (1996)

6. M.I.Dlugach,The grid method in a mixed plain elasticity problem ("NaukovaDumka", Moscow, 1994) 